\title{
In Vivo Validation of Electrocardiographic Imaging
}

Citation for published version (APA):

Cluitmans, M. J. M., Bonizzi, P., Karel, J. M. H., Das, M., Kietselaer, B. L. J. H., Jong, M. M. J. D., Prinzen, F. W., Peeters, R. L. M., Westra, R. L., \& Volders, P. G. A. (2017). In Vivo Validation of Electrocardiographic Imaging. JACC: Clinical Electrophysiology, 3(3), 232 - 242.

https://doi.org/10.1016/j.jacep.2016.11.012

Document status and date:

Published: 01/01/2017

DOI:

10.1016/j.jacep.2016.11.012

Document Version:

Publisher's PDF, also known as Version of record

Document license:

Taverne

Please check the document version of this publication:

- A submitted manuscript is the version of the article upon submission and before peer-review. There can be important differences between the submitted version and the official published version of record.

People interested in the research are advised to contact the author for the final version of the publication, or visit the DOI to the publisher's website.

- The final author version and the galley proof are versions of the publication after peer review.

- The final published version features the final layout of the paper including the volume, issue and page numbers.

Link to publication

\footnotetext{
General rights rights.

- You may freely distribute the URL identifying the publication in the public portal. please follow below link for the End User Agreement:

www.umlib.nl/taverne-license

Take down policy

If you believe that this document breaches copyright please contact us at:

repository@maastrichtuniversity.nl

providing details and we will investigate your claim.
}

Copyright and moral rights for the publications made accessible in the public portal are retained by the authors and/or other copyright owners and it is a condition of accessing publications that users recognise and abide by the legal requirements associated with these

- Users may download and print one copy of any publication from the public portal for the purpose of private study or research.

- You may not further distribute the material or use it for any profit-making activity or commercial gain

If the publication is distributed under the terms of Article $25 \mathrm{fa}$ of the Dutch Copyright Act, indicated by the "Taverne" license above, 


\section{In Vivo Validation of Electrocardiographic Imaging}

Matthijs J.M. Cluitmans, MD, PHD, ${ }^{\mathrm{a}, \mathrm{b}}$ Pietro Bonizzi, PHD, ${ }^{\mathrm{b}}$ Joël M.H. Karel, PHD, ${ }^{\mathrm{b}}$ Marco Das, MD, PHD, ${ }^{c}$ Bas L.J.H. Kietselaer, MD, PHD, ${ }^{\mathrm{a}, \mathrm{c}}$ Monique M.J. de Jong, ${ }^{\mathrm{a}}$ Frits W. Prinzen, PHD, ${ }^{\mathrm{d}}$ Ralf L.M. Peeters, PHD, Ronald L. Westra, PHD, ${ }^{b}$ Paul G.A. Volders, MD, $\mathrm{PH}^{\mathrm{a}}$

\section{ABSTRACT}

OBJECTIVES The purpose of this study was to evaluate the accuracy of noninvasive reconstructions of epicardial potentials, electrograms, activation and recovery isochrones, and beat origins by simultaneously performing electrocardiographic imaging (ECGI) and invasive epicardial electrography in intact animals.

BACKGROUND Noninvasive imaging of electrical potentials at the epicardium, known as ECGI, is increasingly applied in patients to assess normal and abnormal cardiac electrical activity.

METHODS Body-surface potentials and epicardial potentials were recorded in normal anesthetized dogs. Computed tomography scanning provided a torso-heart geometry that was used to reconstruct epicardial potentials from body-surface potentials.

RESULTS Electrogram reconstructions attained a moderate accuracy compared with epicardial recordings (median correlation coefficient: 0.71 ), but with considerable variation (interquartile range: 0.36 to 0.86 ). This variation could be explained by a spatial mismatch (overall resolution was $<20 \mathrm{~mm}$ ) that was most apparent in regions with electrographic transition. More accurate derivation of activation times (Pearson R: 0.82), recovery times (R: 0.73), and the origin of paced beats (median error: $10 \mathrm{~mm}$; interquartile range: 7 to $17 \mathrm{~mm}$ ) was achieved by a spatiotemporal approach that incorporates the characteristics of the respective electrogram and neighboring electrograms. Reconstruction of beats from repeated single-site pacing showed a stable localization of origin. Cardiac motion, currently ignored in ECGI, correlates negatively with reconstruction accuracy.

CONCLUSIONS ECGI shows a decent median accuracy, but variability in electrogram reconstruction can be sizable. At present, therefore, clinical interpretations of ECGI should not be made on the basis of single electrograms only. Incorporating local spatiotemporal characteristics allows for accurate reconstruction of epicardial activation and recovery patterns, and beat origin localization to a $10-\mathrm{mm}$ precision. Even more reliable interpretations are expected when the influences of cardiac motion are accounted for in ECGI. (J Am Coll Cardiol EP 2017;3:232-42) (c) 2017 by the American College of Cardiology Foundation.

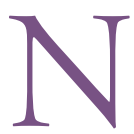
oninvasive electrocardiographic imaging (ECGI) reconstructs epicardial potentials, electrograms, and activation/recovery isochrones from body-surface electrograms (1) (Figure 1A). It is built on the relation between potentials at the heart surface and on the torso, dictated by the laws of electromagnetism. Inverting this relation enables the reconstruction of epicardial potentials from electrocardiograms recorded at the body surface. This requires a thorough understanding

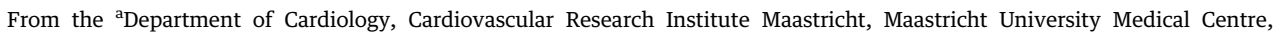
Maastricht, the Netherlands; ${ }^{\mathrm{b}}$ Department of Data Science and Knowledge Engineering, Maastricht University, Maastricht, the Netherlands; 'Department of Radiology, Cardiovascular Research Institute Maastricht, Maastricht University Medical Centre, Maastricht, the Netherlands; and the ${ }^{\mathrm{d} D e p a r t m e n t ~ o f ~ P h y s i o l o g y, ~ C a r d i o v a s c u l a r ~ R e s e a r c h ~ I n s t i t u t e ~ M a a s t r i c h t, ~ M a a s t r i c h t ~ U n i-~}$ versity Medical Centre, Maastricht, the Netherlands. Dr. Volders was supported by the Netherlands Heart Foundation (NHS2007T51) and a Vidi grant from the Netherlands Organization for Scientific Research (ZonMw 91710365). Dr. Cluitmans was supported by the Province of Limburg, the Netherlands (tUL project NS1b). All other authors have reported that they have no relationships relevant to the contents of this paper to disclose.

All authors attest they are in compliance with human studies committees and animal welfare regulations of the authors' institutions and Food and Drug Administration guidelines, including patient consent where appropriate. For more information, visit the JACC: Clinical Electrophysiology author instructions page. 
of the heart-torso relation made on the basis of the patient-specific geometry and conductivity. Anatomical input is mostly provided by computed tomography (CT) or magnetic resonance imaging. In addition, mathematical methods are necessary to overcome the uncertainties that emerge when assessing epicardial potentials from their attenuated and superimposed projections on the torso. A recent review addresses these technical aspects and their clinical implications (2).

ECGI has been used to noninvasively characterize normal activation and recovery in healthy human subjects (3). A main focus has also been to detect the origin of ventricular or atrial tachyarrhythmias, thus expediting diagnostic catheter-based electrophysiology studies and potentially promoting the successful outcome of ablation (4). ECGI of atrial fibrillation (AF) has helped to reduce invasive procedural time during ablation (5). Noninvasive mapping with ECGI provided detailed electrical activation patterns of the left (LV) and right ventricles (RV), which could improve resynchronization therapy (6). Likewise, ECGI can benefit risk stratification for sudden cardiac death by imaging activation and recovery abnormalities as important arrhythmogenic substrates $(7,8)$.

Considering all of these applications, it is remarkable that invasive validation studies of ECGI in intact organisms have been limited. Previous investigations were performed using analytical and computer models (9) and computer models with partially real data (8), or were made on the basis of torso-tank experiments with isolated canine hearts (10) and to some extent experiments in humans (11-13). Each of these studies had distinct advantages, such as the amount of control in the torso-tank experiments and the potential of clinical application in the human studies; however, none of these studies compared noninvasive reconstructions with simultaneous invasive electrograms in an intact, closed-chest organism. For the present study, we examined the reconstruction accuracy and capabilities of ECGI with highprecision simultaneous heart and torso recordings from uniquely instrumented anesthetized dogs.

\section{METHODS}

For a detailed description of the methods, see the Online Appendix.

ANIMAL EXPERIMENTS. This investigation conformed to the Guide for the Care and Use of Laboratory Animals published by the U.S. National Institutes of Health (National Institutes of Health Publication
85-23, revised 1996). Animal handling was in accordance with the European Directive for the Protection of Vertebrate Animals Used for Experimental and Other Scientific Purposes (86/609/EU) and was approved by the institutional review committee for animal studies.

In 4 normal anesthetized dogs, 2 silicone bands with 99 electrodes were implanted around the basal and mid-basal ventricular epicardium after thoracotomy. The position of the epicardial electrodes can be observed in Figure 2. Each band consisted of 2 rows of electrodes. Additional electrodes were placed at the LV's apical epicardium, the LV endocardium, the RV apical endocardium, and the right atrial endocardium, providing a total of 103 electrodes. After chest closure, body-surface electrodes (184 to 216, depending on torso size) were attached to the chest. Reference electrodes were attached on the abdomen near the right lower paw. Unipolar potential recordings were obtained simultaneously by the epicardial and body-surface electrodes. Recordings were performed on the CT table to avoid a change in the geometry or disconnection of the electrodes by moving the animals. A helical electrocardiography (ECG)-gated CT scan was performed with intravenous iodine contrast medium and a diastolic reconstruction of the torso-heart geometry was performed.

INVERSE RECONSTRUCTION. Inverse reconstruction of epicardial potentials was identical to the human application illustrated in Figure 1A and is performed on the basis of the potential-based formulation of ECGI. This formulation assumes that there is a numerical relation between electrical potentials at the heart and body surface (2). It is currently the most used formulation and is available in commercial setups.

Torso-heart geometry was digitized from the CT scan and contained the body-surface electrodes and the ventricular epicardial surface (consisting of on average 1,693 nodes; mean node-to-node distance: $4 \mathrm{~mm}$ ). Additionally, the position of the 103 implanted electrodes was digitized.

Inverse reconstruction of epicardial potentials from the recorded body-surface potentials is subject to uncertainty because of attenuating and dispersing effect of the electromagnetic propagation from heart to body surface (2). As a consequence, most implementations of the inverse problem are strongly affected by noise in the body surface signals and approximations in the heart-torso geometry, and require mathematical regularization methods to obtained physiologically meaningful reconstructions.
ABBREVIATIONS AND ACRONYMS

AF $=$ atrial fibrillation $\mathrm{C}=$ correlation coefficient $\mathrm{T}=$ computed tomography $V / d t=$ derivative of potential EGI = electrocardiographic maging $Q R$ = interquartile range $\mathrm{V}=$ left ventricle $\mathrm{V}=$ right ventricle 
FIGURE 1 ECGI Noninvasively Reconstructs Electrograms and Activation and Recovery Isochrones on the Epicardium, Which Were Evaluated in Canine Experiments

A Electrocardiographic imaging

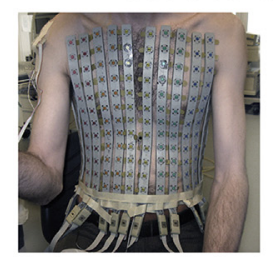

Body-surface electrodes

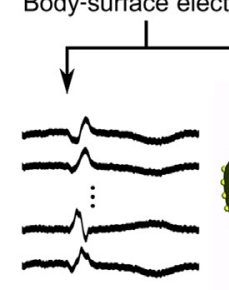

ECGs

Torso with potentials

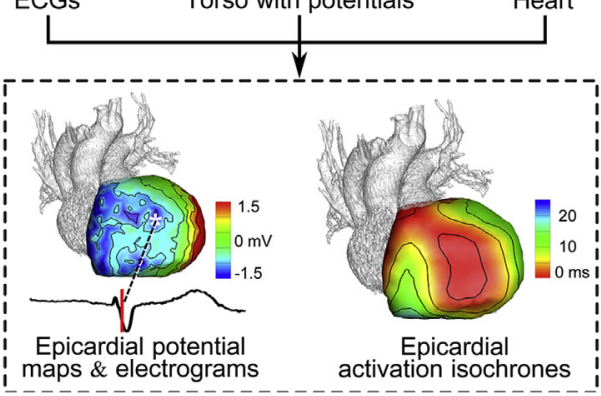

B Accuracy of noninvasive reconstruction
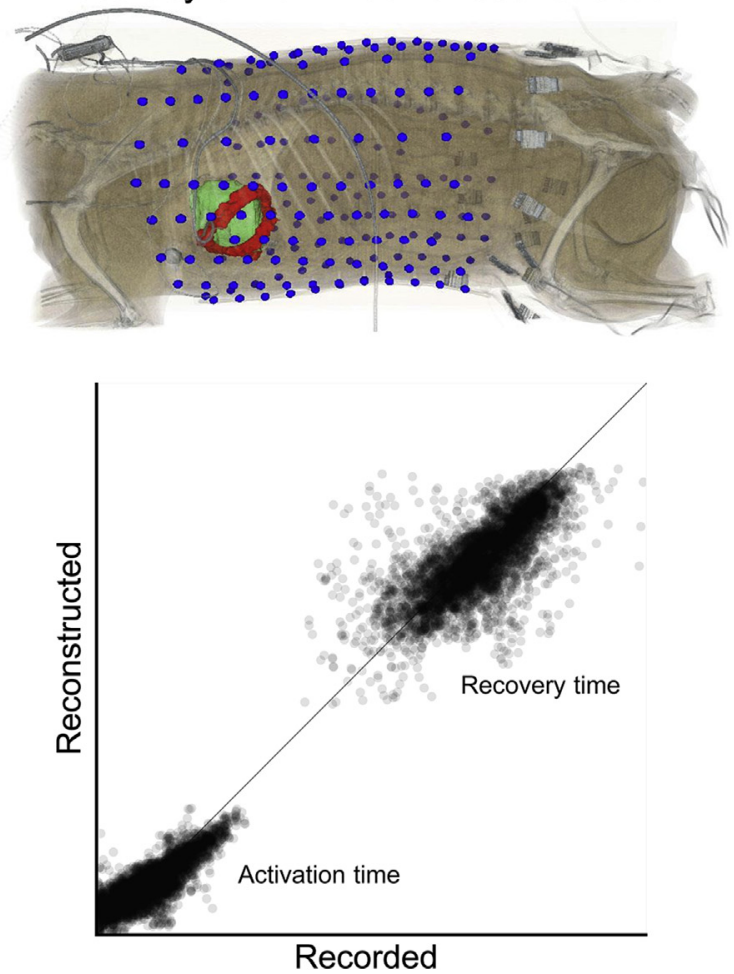

(A) Noninvasive electrocardiographic imaging (ECGI) as it is generally applied in humans. Body-surface ECGs are combined with a torso-heart geometry obtained with computed tomography scans. By carefully reversing models of the physical laws of electromagnetism, epicardial potentials can be reconstructed. From these, epicardial electrograms and isochrones are deducted. (B) The experimental setup as applied in a normal anesthetized dog in the validation experiments of this study, illustrating the body-surface electrodes (blue), the epicardial surface (green), and the epicardial electrodes (red). The graph shows that activation and recovery times are accurately reconstructed with ECG imaging.

Commonly used methods were applied to address this in our study, to reconstruct epicardial potentials (2). We refer to "nodes" as the virtual points on the epicardial surface on which potentials are reconstructed, and to "electrodes" as the physically implanted electrodes that record epicardial potentials.

POST-PROCESSING TO OBTAIN ELECTROGRAMS, ISOCHRONES, AND ACTIVATION ORIGIN. After reconstruction of epicardial potentials, electrograms were reconstructed per node by concatenating potentials over time. Activation times were determined per electrogram with 2 methods: the temporal-only and a spatiotemporal method. The temporal-only approach defines the moment of activation as the moment of steepest voltage downslope (maximum derivative of potential $[-d V / d t]$ ) during the QRS complex. The spatiotemporal approach, proposed by Erem et al. (14), takes advantage of the spatial relationship between neighboring nodes and their potentials and may be better suited to estimate the activation time in noisy or fractionated electrograms. This approach selects the moment that matches the change in temporal derivative with the change in spatial derivative (14). Recovery times were defined as the moment of maximum $d V / d t$ during the electrographic T-wave.

All reconstructions and analyses were performed automatically to prevent bias by the researchers and investigate the robustness and practicality of the technique.

STATISTICAL ANALYSIS. For each epicardial electrode, Pearson correlation coefficient (CC) was computed between the recorded potentials and the reconstructed potentials at the corresponding (closest) virtual epicardial node. CCs computed over an electrogram are called temporal CCs (and mean 
A
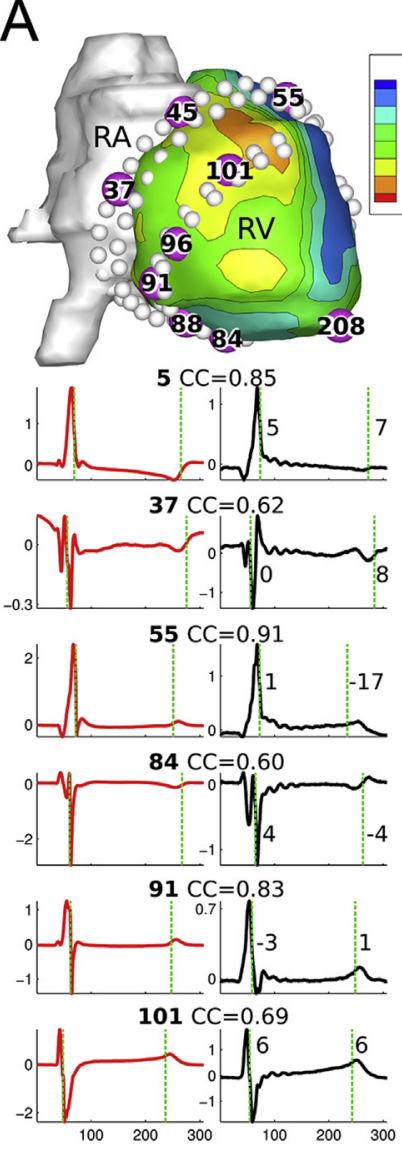

B
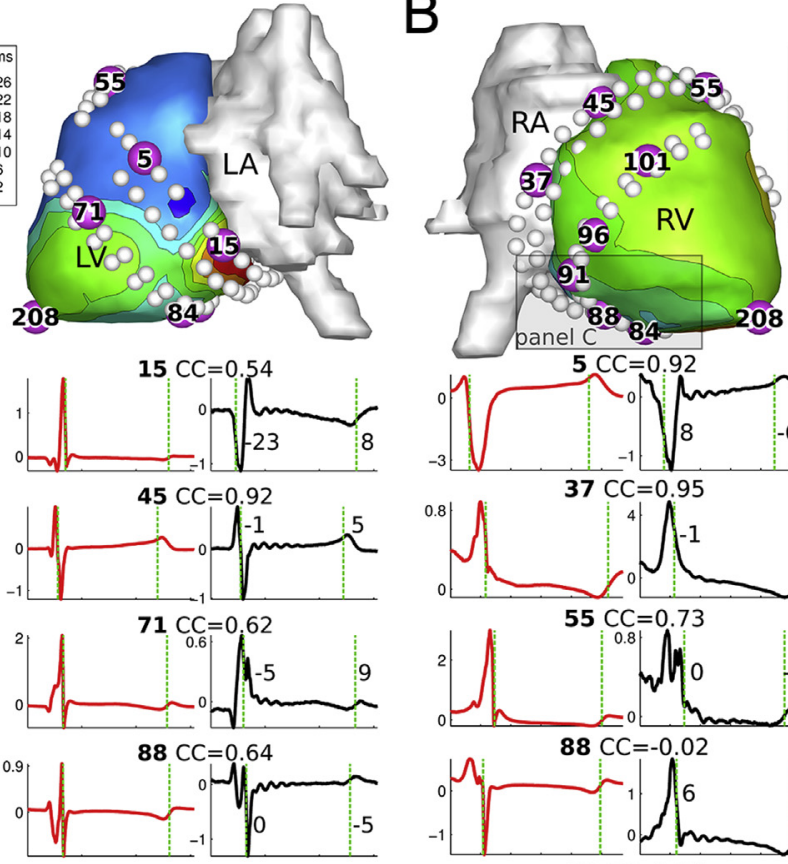

$96 \mathrm{CC}=0.80$
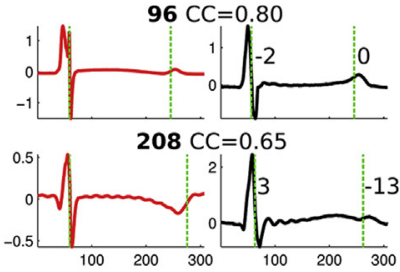
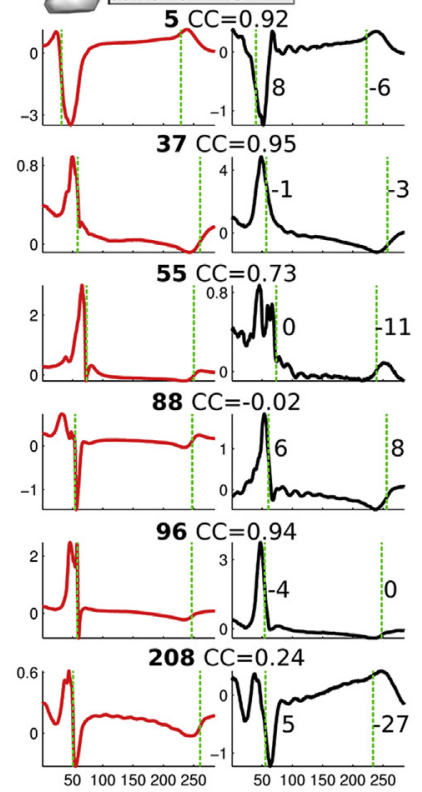
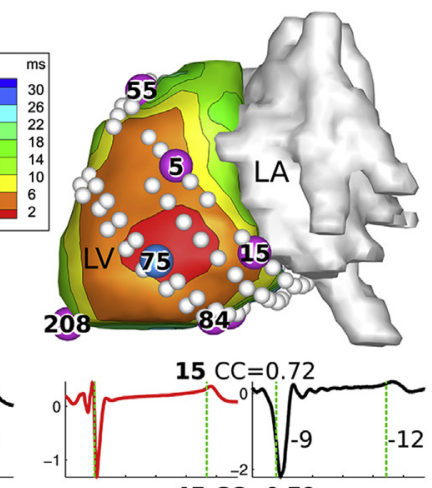

$45 \mathrm{CC}=0.79$
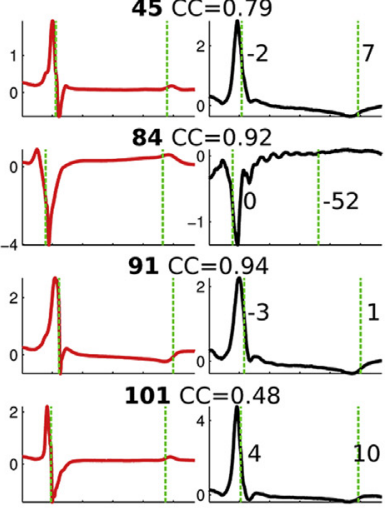

${ }_{\mathrm{ms}}^{\mathrm{mV}}$

C<smiles>CCCCCC</smiles>

$\leftarrow \stackrel{\frac{0}{n}}{\circ}$<smiles>C1CC1</smiles><smiles>CC[Te]</smiles>

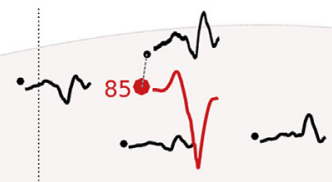<smiles>C1CCCC1</smiles><smiles>C1CCCCC1</smiles><smiles>[Te]=[Te]</smiles>

86<smiles>C1CCCCC1</smiles>

in

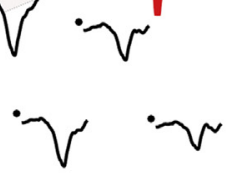

$-$

Negative polarity in recording and reconstruction: high correlation
Negative polarity in recording and positive polarity in reconstruction: low correlation

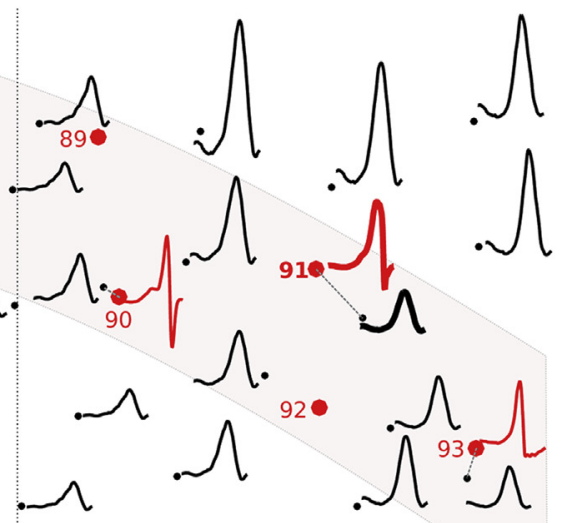

$++$

Positive polarity in recording and

Spheres at the heart surface indicate the position of epicardial electrodes. For the numbered purple spheres, the recorded (red, left) and reconstructed (black, right) electrograms are depicted below. For each pair of electrograms, the correlation coefficient (CC) is given. Additionally, activation and recovery times are indicated with vertical green lines. The mismatch between the recorded and reconstructed time is given in milliseconds at the right of each pair. The epicardial colors indicate noninvasive ventricular activation isochrones. (A) Sinus beat; (B) beat paced at left ventricular epicardial electrode 75 (blue sphere). (C) Magnification of the region of electrodes 84-88-91 of the paced beat (only QRS complex shown), showing that the polarity change in the recorded electrograms (red) is more distal of the pacing site then in the reconstructed electrograms (black). $L A=$ left atrium; $L V=$ left ventricle; $R A=$ right atrium; $R V=$ right ventricle. 
temporal CCs are averaged over the electrograms of all electrodes); CCs computed over spatial distributions of potentials are called spatial CCs (and mean spatial CCs are averaged of all time instants of a recording). Linear correlation between measured and reconstructed activation/recovery timings was assessed by means of Pearson correlation R. Statistical comparison of correlation coefficients and activation and recovery timings was achieved by Wilcoxon signed rank (for paired measurements) or Wilcoxon rank sum (for unpaired measurements) tests.

\section{RESULTS}

In the 4 dogs, 140 beats were analyzed: 93 morphologically unique beats (in which only 1 beat per pacing site was included) and 47 additional non-unique beats (repeated single-site pacing) at 4 different locations. In the 93 unique beats, on average 60 epicardial electrodes provided high-quality potential recordings, resulting in 5,552 pairs of recorded and reconstructed electrograms. A large diversity of beats was analyzed: normally conducted sinus beats $(\mathrm{n}=6$ beats), atrially paced beats with normal ventricular activation $(\mathrm{n}=7)$, and beats paced from the $\mathrm{LV}$ endocardium $(n=3)$, RV endocardium $(n=2)$, ventricular epicardium $(\mathrm{n}=71)$, or by biventricular epicardial pacing $(n=4)$. The additional 47 beats occurred upon repeated pacing at 4 different locations to determine the stability of origin localization.

ACCURACY OF RECONSTRUCTED ELECTROGRAMS. Figure 2 shows representative examples of recorded and reconstructed electrograms for a sinus beat (panel A) and a LV epicardially paced beat (panel B). Median correlation coefficient calculated for the entire signals was 0.71 for all 5,552 pairs of recorded and reconstructed electrograms. There was a considerable (skewed) spread in correlation coefficients, resulting in an interquartile range (IQR, spanning the $25 \%$ to $75 \%$ range of data) of 0.36 to 0.86 . Of the electrograms with a correlation coefficient $<0.40$, $65 \%$ was found in a region with changing electrogram morphologies, as illustrated in panel $\mathrm{C}$ for electrode 88 of the paced beat (computational details in the Online Appendix).

Figure 2 also shows activation and recovery times in the recorded and reconstructed electrograms, defined as the moment of maximum $-d V / d t$ (in the QRS complex) and maximum $d V / d t$ (in the T-wave), respectively. The sinus beat (Figure $2 \mathrm{~A}$ ) shows early epicardial breakthrough at the anterior RV. The paced beat (Figure 2B) shows early activation at the location of pacing on the $\mathrm{LV}$, and consequently late activation of the RV. For locations on the epicardium for which recorded electrograms were available, generally there was an activation time mismatch of only a few milliseconds, unless the reconstructed electrogram deviated considerably from the recorded one (e.g., electrode 15 in Figure 2A). Overall, recovery times had a larger error than activation times.

Figure 3A shows the temporal CC for different locations on the heart: LV anterior, lateral, posterior, RV lateral, and LV apical epicardium, respectively. Medians for the $\mathrm{LV}$ anterior and RV lateral regions were significantly different from the other regions ( $\mathrm{p}<0.05$ ); however, the large overlap in data suggests that this is mainly an effect of the large sample size and has limited practical value. The CC at the apex, determined from only a single electrode and not an ensemble of electrodes as with the other regions, was significantly higher than at the other regions.

Figure 3B shows the temporal CC for the entire QRST complex and its different segments. Again, these were statistically significantly different, although, because of the large overlap, these differences will have limited clinical value.

Figure $3 \mathrm{C}$ depicts the average spatial CC between the recorded and reconstructed potentials for all epicardial electrodes, averaged for all 93 beats. In general, the spatial CC decreased at the end of the QRS complex and the end of the T-wave.

In Figure 3D, spatial resolution of reconstructed electrograms is depicted. For each recorded electrogram, the distance was calculated between the recording electrode and the closest epicardial node that resulted in a good-enough correlation (defined as CC $>0.70$ ). For $84 \%$ of all recorded electrograms, a good-enough CC was found with a reconstructed electrogram at some location on the heart surface, and the distance to the closest epicardial node resulting in a good-enough correlation was $<20 \mathrm{~mm}$ in $90 \%$ of these cases.

\section{ACCURACY OF RECONSTRUCTED ACTIVATION AND} RECOVERY TIMES. Figure 3E shows the association between timings determined from the recorded and from the reconstructed electrogram. Activation times as determined with the spatiotemporal approach (red dots, $\mathrm{R}=0.82$ ) showed a stronger association $(\mathrm{p}<0.05)$ than those determined with the temporal approach (gray dots in the background of the red dots, $\mathrm{R}=0.73$ ). Recovery times were significantly more accurately $(\mathrm{p}<0.05)$ determined after spatial smoothing (blue dots, $\mathrm{R}=0.73$ ) than without (gray dots, $\mathrm{R}=0.60$ ). Activation times could be determined more accurately than recovery times. 
FIGURE 3 Accuracy of Noninvasive Reconstruction of Epicardial Potentials
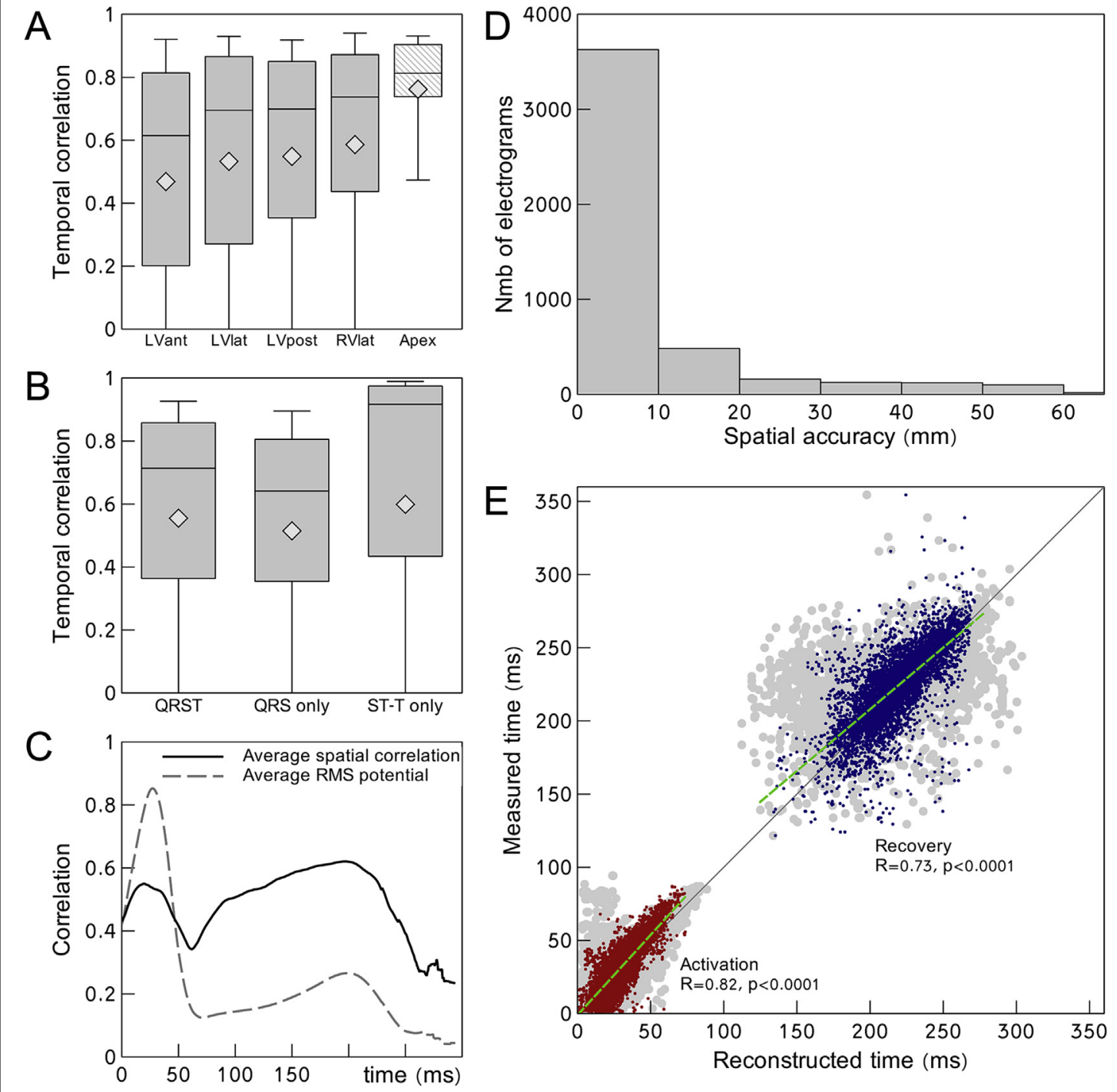

(A) Temporal correlation coefficients for different locations on the heart: LV anterior (LVant)/lateral (LVlat)/posterior (LVpost) sides, RV lateral side (RVlat; all on the basis of multiple electrodes), and RV apex (single electrode). (B) Temporal correlation coefficients for the full QRST segment of the cardiac beat (left), for only the QRS complex (middle), and only the ST-T segment (right) of all beats, respectively. (C) Spatial correlation coefficient per time instant (solid line), averaged over all electrograms. The root mean square (RMS) of the recorded epicardial potentials shows the average moment of the QRS complex (first peak) and T wave (second peak). (D) Histogram for recorded electrograms, showing the distance between the recording electrode and the closest virtual epicardial node, which resulted in a good-enough correlation (CC >0.70). (E) Activation (red dots) and recovery (blue dots) times as determined with the spatiotemporal approach from recorded electrograms (vertical axis) versus the timings determined from reconstructed electrograms (horizontal axis); gray dots in the background show the times as determined with the less accurate temporal-only approach. Timings are relative to QRS onset. Data collected over 93 beats in the 4 dogs (5,552 pairs of electrograms). Abbreviations as in Figure 2.

LOCALIZATION OF BEAT ORIGIN. One major application of ECGI has been its ability to localize the origin of ventricular extrasystolic beats and focal tachycardia. We have investigated this by noninvasively reconstructing the origin of beats that were paced by ventricular electrodes. The reconstructed location of earliest activation was compared with the known location of ventricular pacing for 80 beats in the 4 dogs. Each pacing location was included only once. Figure 4A shows that the median error of the temporal-only approach for activation timing was $33 \mathrm{~mm}$, with a range of 5 to $79 \mathrm{~mm}$. 
FIGURE 4 Accuracy of Noninvasive Localization of the Origin of Paced Beats
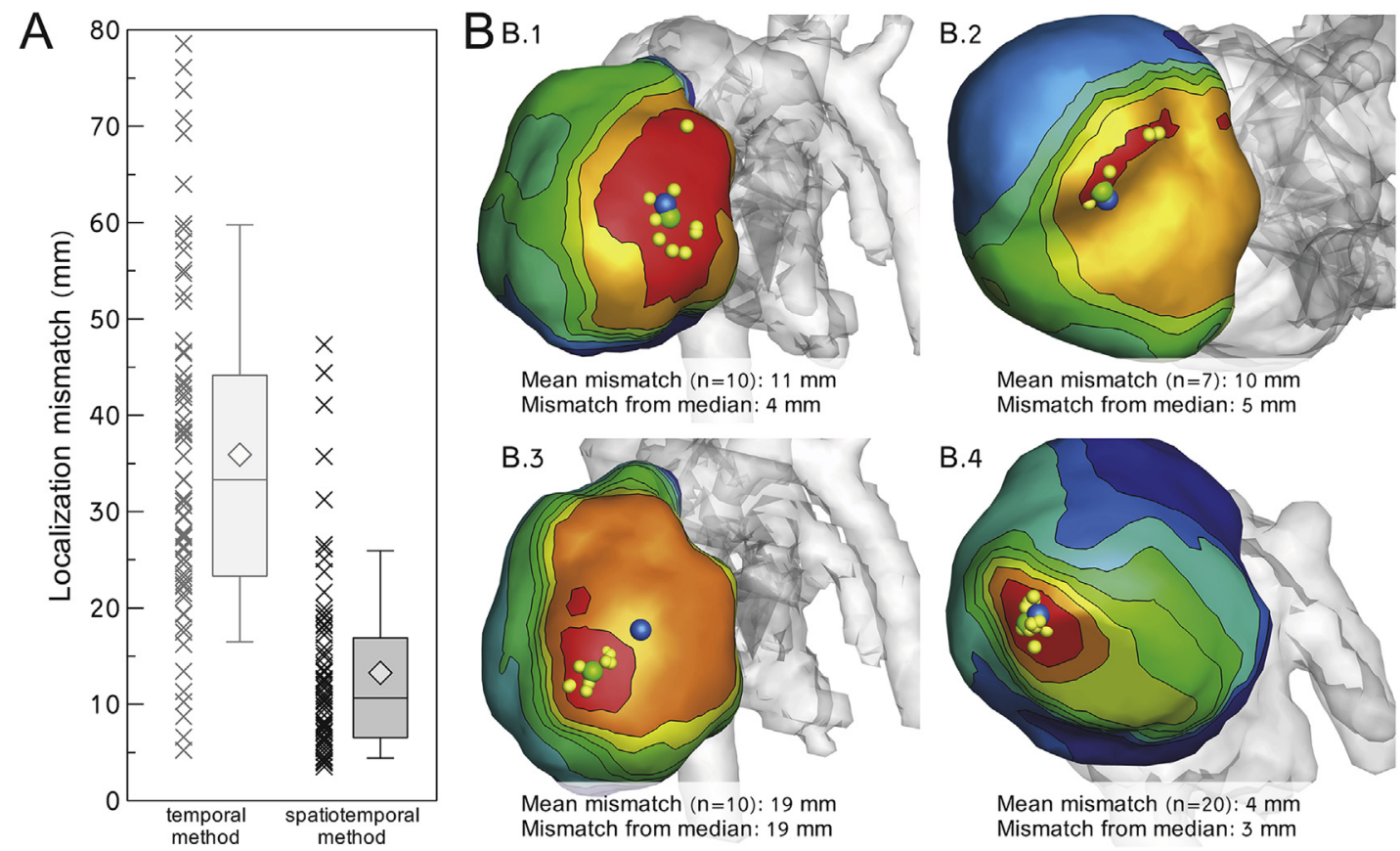

Mean mismatch $(n=7): 10 \mathrm{~mm}$

Mismatch from median: $5 \mathrm{~mm}$
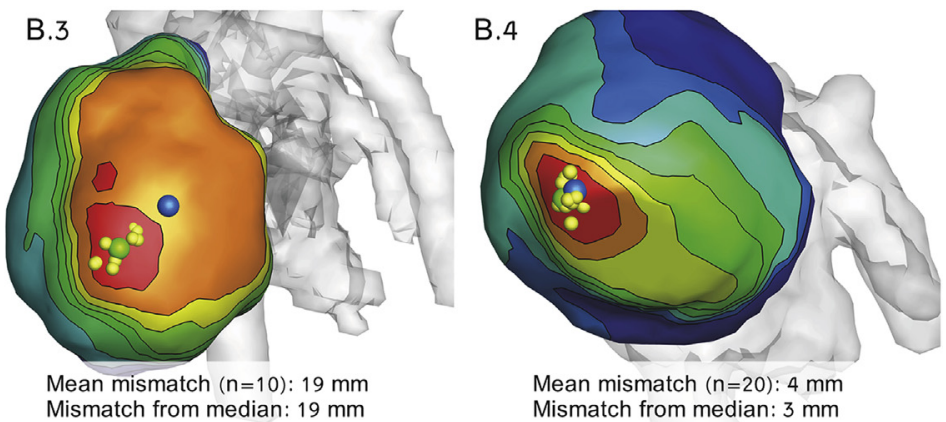

(A) Scatter plots and box plots for localization mismatch with 2 methods (temporal-only and spatiotemporal method) for 80 paced beats in 4 dogs. Localization mismatch is defined as the distance between the known pacing location and the location of earliest activation from noninvasively reconstructed epicardial electrograms. (B) Reconstructed origins of consecutive beats paced at the location indicated with the blue dot. Epicardial surface color indicates the reconstructed activation time for the first beat of that series (red: 0 ms, isochrone lines drawn at 5 -ms intervals). Yellow dots indicate the reconstructed origins for all beats of that series, all paced at the same location. The green dot is the median location of earliest activation and usually has a lower mismatch with the known pacing location than the mismatch of all beats.

These results improved significantly by using the spatiotemporal approach, which resulted in a median error of only $10 \mathrm{~mm}$ (range: 3 to $47 \mathrm{~mm}$; $\mathrm{p}<0.05)$. The range of errors decreased considerably: for the temporal-only approach, the origins of $90 \%$ of the beats lay within a $60-\mathrm{mm}$ range from the known pacing location, whereas this range decreased drastically to $25 \mathrm{~mm}$ with the spatiotemporal approach.

We also analyzed reconstructions of beats paced repeatedly at the same location to investigate the stability of detection of the origin of these beats. For this purpose, 4 different pacing sites were investigated, with a total 47 additional beats (these beats were not included in the previous analysis to avoid bias) (Figure 4B). In general, all the detected origins (yellow spheres) for the same beat were within a confined region around the pacing location (blue sphere). Moreover, the median location (green sphere) of all detected origins had a lower mismatch than the mean mismatch of individual detected origins.

\section{CARDIAC MOVEMENT AND RECONSTRUCTION} ACCURACY. Please refer to the Online Appendix for the analysis of cardiac movement and reconstruction accuracy to see that the reconstruction accuracy is smaller when motion is larger. The Online Appendix also provides an analysis of the endocardial or epicardial origin of paced beats (which is clinically relevant to select the correct ablation approach), examples of canine cardiac activation and recovery patterns, and a link to our freely shared data to stimulate further research.

\section{DISCUSSION}

Noninvasive ECGI is increasingly applied in clinical settings. Table 1 summarizes recent validation studies that evaluated ECGI with quantitative data (e.g., from artificial torso tank setups to non-simultaneous invasive recordings in humans). In the present study, we acquired simultaneous recordings of bodysurface and invasive epicardial potentials in normal anesthetized dogs under closed-chest conditions to 


\begin{tabular}{|c|c|c|c|c|c|c|c|}
\hline Medium and Method & $\begin{array}{l}\text { No. of } \\
\text { Subjects }\end{array}$ & $\begin{array}{l}\text { No. of } \\
\text { Beats }\end{array}$ & $\begin{array}{l}\text { Mean Electrogram } \\
\text { Correlation }\end{array}$ & $\begin{array}{c}\text { Mean Pacing } \\
\text { Localization Error }(\mathrm{cm})\end{array}$ & $\begin{array}{c}\text { Correlation of } \\
\text { Activation Times }\end{array}$ & $\begin{array}{l}\text { Correlation of } \\
\text { Recovery Rimes }\end{array}$ & Reference \\
\hline \multicolumn{8}{|l|}{ Torso tank } \\
\hline Single-site epicardial pacing in torso tank & - & - & - & $<1.0$ & Qal & - & 10 \\
\hline Single- and dual-site epicardial pacing in torso tank & - & 4 & 0.81 & 0.2 & Qal & - & 15 \\
\hline \multicolumn{8}{|l|}{ Humans } \\
\hline $\begin{array}{l}\text { Intraoperative mapping in patients while pacing } \\
\text { (nonsimultaneous recording, open chest) }\end{array}$ & 3 & 5 & $0.72 \pm 0.25^{*}$ & $\sim 1.0$ & Qal & - & 11 \\
\hline Ventricular pacing by implanted pacemaker & 4 & 6 & - & 0.5 & - & - & 15 \\
\hline Endocardial atrial pacing in AF patients & 6 & 37 & - & $0.6 \pm 0.4$ & - & - & 12 \\
\hline Epicardial ventricular pacing & 4 & 79 & - & $1.3 \pm 0.9$ & Qal & - & 13 \\
\hline Pacemaker atrial/ventricular pacing & 29 & $456+$ & - & $0.9 \pm 0.6$ & - & - & 16 \\
\hline Endocardial atrial/ventricular pacing & 5 & $412 \dagger$ & - & $0.7 \pm 0.2$ & - & - & 16 \\
\hline \multicolumn{8}{|l|}{ Canines } \\
\hline Epicardial and endocardial ventricular pacing & 4 & 93 & $0.71[0.36-0.86] \ddagger$ & $1.0[0.7-1.7] \ddagger$ & 0.82 & 0.73 & Present study \\
\hline \multicolumn{8}{|c|}{$\begin{array}{l}\text { Overview of recent in vivo validation studies of the potential-based problem of ECGI. Only studies providing quantitative data (i.e., pacing location mismatch or invasive electrogram comparison) on ECGI } \\
\text { validation were included. *Determined by allowing a time-shift (cross-correlation). IIncludes beats paced from identical locations (non-unique morphology). } \neq(\text { Median [IQR]). }\end{array}$} \\
\hline \multicolumn{8}{|c|}{$\mathrm{AF}=$ atrial fibrillation; $\mathrm{ECGI}=$ electrocardiographic imaging; IQR = interquartile range; Qal = qualitative comparison, no quantitative comparison. } \\
\hline
\end{tabular}

compare reconstructed with recorded epicardial electrograms, activation/recovery timings, and beatorigin localization.

VARIABLE ACCURACY OF RECONSTRUCTED ELECTROGRAMS. Direct comparison of reconstructed and recorded electrograms showed that, in general, noninvasively reconstructed epicardial electrograms correlated well with the invasively recorded signals, with an overall median CC for all signals of 0.71. However, single electrograms could be discrepant, resulting in a lower CC. For $90 \%$ of all recorded electrograms for which direct comparison to the closest node showed a suboptimal correlation (defined as CC <0.70), a good-enough match (CC $>0.70$ ) was found within a $20-\mathrm{mm}$ region and $77 \%$ within a $10-\mathrm{mm}$ region. These results suggest that low correlations are primarily caused by a spatial mismatch between reconstructed and recorded electrograms and that this spatial shift is generally small but important.

Our findings are in agreement with previous studies (Table 1) and extend these results on several levels. Previous torso-tank experiments, with explanted canine hearts suspended in a container with electrolyte solution, showed a mean CC for observed and reconstructed signals ( $>4$ beats) of 0.81 (15). The higher accuracy of that study might be explained by the lack of torso inhomogeneities (such as lungs), no breathing motion artifacts, and the distance of the recording electrodes to the epicardium (approximately $1 \mathrm{~cm}$ ). In a previous human study, non-simultaneous invasive recordings during openheart surgery were compared with preprocedural noninvasive reconstructions (11). These investigators showed a mean cross correlation of $0.72 \pm 0.25$ over 5 beats in 3 subjects. "Cross correlation" is defined by shifting the reconstructed electrogram over the recorded one and computing the maximum correlation coefficient during this process. The results of that study should thus be compared to our "timeshifted" correlation coefficients, as presented in the Online Appendix. The mean values of time-shifted CCs $\left(\mathrm{CC}_{\text {mean }}=0.72\right)$ by Ghanem et al. (11) are comparable with ours $\left(\mathrm{CC}_{\text {mean }}=0.71\right)$. Our study extends these previous results by reporting medians and ranges and by examining many more beats under closed-chest conditions (i.e., much closer to the noninvasive clinical situation).

Moreover, in the Online Appendix we show that there is a negative association between cardiac movement and reconstruction accuracy. This should be investigated further; it is to be expected that incorporating cardiac movement in ECGI will improve accuracy of reconstructed electrograms.

\section{SPATIOTEMPORAL APPROACH ENHANCES} RECONSTRUCTION ACCURACY. Accuracy of activation has been evaluated previously by qualitatively comparing activation isochrones in torso tank experiments and human studies (Table 1). To the best of our knowledge, such an evaluation does not exist for recovery isochrones. We found that single electrograms could deviate considerably from the true (recorded) electrogram; consequently, activation and recovery times could deviate considerably between reconstruction and recording. Noise, fractionation, and reconstruction inaccuracies can lead to suboptimal results when using temporal-only criteria. By concatenating the characteristics of local 
electrograms as suggested by Erem et al. (14), more robust interpretations were made, and this spatiotemporal approach led to high correlation $(\mathrm{R}=0.82)$ for activation timings. Recovery timings attained slightly less accuracy $(\mathrm{R}=0.73)$, which could be explained by lower amplitudes and less steep slopes of the electrographic $\mathrm{T}$ wave, making it more sensitive to noise compared with the QRS complex. Additionally, when ignoring cardiac motion, the assumption of a static diastolic geometry has its largest negative impact on the reconstruction of the ST-T segment. In conclusion, activation time maps and, to a lesser extent, recovery time maps can be interpreted with confidence if a spatiotemporal algorithm is used.

ECGI-BASED LOCALIZATION OF ECTOPIC BEATS IS ACCURATE WITHIN THE CENTIMETER RANGE. ECGI has been commonly applied to localize the origin of ventricular extrasystolic beats (Table 1). The first study to investigate localization accuracy in humans found a precision of approximately $1 \mathrm{~cm}$, made on the basis of 3 paced beats (11). In another study, ventricular pacing was performed in humans with simultaneous recording of body-surface potentials; inverse reconstruction of the earliest site of activation had a median error of $1.3 \pm 0.9 \mathrm{~cm}$ in healthy hearts (13). In a similar study, an accuracy of $0.9 \pm 0.6$ $\mathrm{cm}$ and $0.7 \pm 0.2 \mathrm{~cm}$ was obtained (16). In atria, pacing sites could be localized with $0.6 \pm 0.4 \mathrm{~cm}$ accuracy (12). In the present study, we have shown that a spatiotemporal approach to determine the site of origin of paced beats resulted in a median error of $1.0 \mathrm{~cm}$. Of importance, the range of errors was limited, with $75 \%$ of the beats having an error $<1.7 \mathrm{~cm}$ and $90 \%<2.5 \mathrm{~cm}$.

During ablation therapy, the location of ablation is confirmed with invasive activation and pace mapping, with an assumed accuracy of several millimeters. Although ECGI is accurate to a centimeter, not millimeter level, it is much more accurate in origin localization than human interpretation of 12-lead electrocardiography (17) and could thus help speeding up ablation procedures by reducing the initial mapping area to an area with a $2-\mathrm{cm}$ radius. This study was the first to investigate the reproducibility of beat localization and to show that noninvasive localization of pacing yields consistent and stable results, which is important for reliable clinical application.

Additionally, ECGI could influence ablation strategies before starting a procedure. Most important, the ability to distinguish between an endocardial or epicardial origin could facilitate the choice between an endocardial vascular or transthoracic epicardial catheter approach, reducing the need to convert from one to the other in specific cases. Although we could not investigate this extensively because of a limited number of endocardial pacing opportunities, Online Figure 3 shows an example in which an endocardial origin on the left ventricle gave an rS morphology on the epicardial electrogram and a Q morphology for an epicardial origin. However, inaccuracies in reconstruction can incorrectly result in small $\mathrm{r}$ deflections suggestive for an endocardial origin, and it is unknown whether the thinner RV wall also gives rise to a detectable $r$ deflection in case of endocardial origins.

The localization error found in this study and others appears low enough for ECGI to support (but not blindly steer) catheter-based diagnostic evaluation, thereby potentially improving therapeutic outcome. However, for completely noninvasive treatment, as suggested recently by Cuculich et al. (18), a more precise localization might be necessary. Currently, these results apply only to healthy hearts and are made on the basis of beats that were mostly paced from the epicardium.

COMPARISON TO OTHER APPROACHES. MANY modeling choices are possible in ECGI. We have aimed at following common choices, but other approaches may yield better results. For example, we have applied Tikhonov zeroth order regularization, whereas other regularization methods may yield improved results in certain situations (e.g., the generalized minimal residual method might better distinguish between multiple pacing sites) (19). A method that uses meshless surfaces has been shown to be more accurate than when triangulated surfaces are used (20), as they were here. Furthermore, it has been shown that beat origin localization could be more accurate not when activation times are used, but rather when the location of deepest negative potential during early activation is used (21). These different approaches in different laboratories illustrate the challenge in comparing and generalizing accuracies.

In this study, we investigated the potential-based formulation of the inverse problem. Originally, it was thought that this method could lead to the reconstruction of epicardial potentials only. Meanwhile, researchers have tried to reconstruct endocardial potentials as well (22). These methods have not yet been investigated extensively or validated. A followup of this validation study could include endocardial potential recordings as well, to evaluate the accuracy of noninvasive reconstructions of endocardial potentials. 
A radically different approach to the inverse problem is the wave-front formulation, which aims to reconstruct activation and recovery times directly without intermediate reconstruction of cardiac potentials (23). If one is only interested in activation and recovery times, this approach would have the theoretical advantage that by skipping the step of reconstruction of electrograms (with its inherent uncertainty), more reliable timings are obtained. However, no in vivo validation studies have been published, and these methods did not yet find widespread clinical application. We plan to compare the potential-based formulation and wave-front formulation in future research.

STUDY LIMITATIONS. By using 2 electrode bands, there was no uniform recording coverage of the ventricles; however, a balance was achieved by positioning these bands at the basal and mid-basal sections of the ventricles (covering the circumferentially largest parts of the ventricles) and an additional electrode at the apex. The single electrode at the apex achieved a significantly higher correlation for reconstructed electrograms than the other epicardial regions. This suggests that our results, which are based mainly on recordings in basal and mid-basal regions, represent the lower bounds of reconstruction accuracy of the full heart.

Implantation of electrodes, contained by silicone bands, could alter local conductivity and propagation of electrical signals to the torso. Bear et al. (24), however, did not find any difference when comparing body-surface electrograms before and after electrode implantation in their recent study.

We investigated a large diversity of rhythms: sinus, atrially paced, and endocardial and epicardial ventricular paced rhythm, with rates ranging from 85 to 143 beats/min. We did not investigate AF or ventricular fibrillation, which is much more complex and most likely more difficult to reconstruct.

To comply with most commonly used ECGI implementations, we have chosen a torso-heart geometry that did not contain any inhomogeneities with distinct conductivity, such as lungs, fatty tissue, and bones. Although these inhomogeneities may alter propagation of electromagnetic fields, their inclusion is time-consuming and increases complexity of the inverse reconstruction, making it more sensitive to noise. In a recent forward study, Bear et al. showed that inclusion of torso inhomogeneities did not improve most of the forward computations (24).

We did not obtain any atrial recordings. Because of the lower potential amplitude of atrial activity on the body surface compared with ventricular activity, reconstruction of atrial epicardial potentials likely is more sensitive to noise. This might be exaggerated in more disorganized, low-amplitude rhythms such as AF. Other studies have suggested that ECGI is able to reconstruct $\mathrm{AF}$ activation patterns, either by direct electrogram reconstruction (12) or by applying socalled "phase mapping” as post-processing (5). Pace origin localization in the atria was shown to be accurate to $6 \mathrm{~mm}$ (12). To the best of our knowledge, no study has compared reconstructed and recorded atrial electrograms, either in normal atrial rhythm or in AF.

\section{CONCLUSIONS}

This validation study addressed the accuracy of electrogram reconstructions for normal and abnormal electrical ventricular activity in intact anesthetized dogs, filling the gap between previous experiments with explanted hearts and ECGI application in humans. Noninvasive reconstruction of epicardial potentials is generally attained at a decent median accuracy, but with considerable spread. Generally, the spatial resolution was far smaller than $20 \mathrm{~mm}$. Results on activation and recovery timing were most reliable when the characteristics of neighboring electrograms were incorporated as well, allowing for accurate and consistent reconstruction of single beat origins (median error: $10 \mathrm{~mm}$; 90\% within $25 \mathrm{~mm}$ ). Upon translation, this supports adequate applications of ECGI at the ventricles during regular and abnormal rhythm, which could expedite catheter-based diagnostic evaluation and ablation in clinical settings. Accurate reconstruction of activation and recovery isochrone maps, as validated in this study, will help improve understanding of (and risk stratification for) cardiac arrhythmias in future applications.

ACKNOWLEDGMENTS The authors thank Roel Spätjens, BSc, for help with the figures; and Lars van Middendorp, MD, PhD; Marc Strik, MD, PhD; and Marion Kuiper, BSc, for their invaluable support in the animal experiments. Furthermore, the authors thank Dana Brooks, PhD; Jaume Coll-Font, MSc (Northeastern University, Boston, Massachusetts); and Burak Erem, PhD (Harvard Medical School and Boston Children's Hospital, Massachusetts) for kindly sharing their algorithm for spatiotemporal activation time approximation.

ADDRESS FOR CORRESPONDENCE: Dr. Paul G.A. Volders, Department of Cardiology, Cardiovascular Research Institute Maastricht, Maastricht University Medical Centre, P.O. Box 5800, 6202 AZ Maastricht, the Netherlands. E-mail: p.volders@ maastrichtuniversity.nl. 


\section{PERSPECTIVES}

COMPETENCY IN MEDICAL KNOWLEDGE: Noninvasive imaging of activation and recovery isochrones is accurate compared with invasive measurements.

TRANSLATIONAL OUTLOOK 1: Noninvasive imaging of activation/recovery isochrones might yield important patient-specific insights in arrhythmia mechanisms.
TRANSLATIONAL OUTLOOK 2: Noninvasive electrocardiographic imaging might be improved further when cardiac and thoracic motions are accounted for.

TRANSLATIONAL OUTLOOK 3: Noninvasive localization of ventricular extrasystolic beats could guide catheter-based therapy and improve clinical outcome; this should be studied in a randomized controlled trial.

\section{REFERENCES}

1. Ramanathan C, Ghanem RN, Jia P, Ryu K, Rudy Y. Noninvasive electrocardiographic imaging for cardiac electrophysiology and arrhythmia. Nat Med 2004;10:422-8.

2. Cluitmans MJM, Peeters RLM, Westra RL, Volders PGA. Noninvasive reconstruction of cardiac electrical activity: update on current methods, applications and challenges. Neth Heart J 2015;23:301-11.

3. Ramanathan $C$, Jia $P$, Ghanem R, Ryu K, Rudy $Y$. Activation and repolarization of the normal human heart under complete physiological conditions. Proc Natl Acad Sci USA 2006;103:6309-14.

4. Shah AJ, Hocini M, Xhaet O, et al. Validation of novel 3D electrocardiographic mapping of atrial tachycardias by invasive mapping and ablation: multicenter study. J Am Coll Cardiol 2013;62 889-97.

5. Haissaguerre $M$, Hocini $M$, Denis $A$, et al. Driver domains in persistent atrial fibrillation. Circulation 2014;130:530-8.

6. Varma $N$, Jia $P$, Ramanathan $C$, Rudy $Y$. RV electrical activation in heart failure during right left, and biventricular pacing. J Am Coll Cardiol Img 2010;3:567-75.

7. Vijayakumar R, Silva JNA, Desouza KA, et al. Electrophysiologic substrate in congenital long QT syndrome: noninvasive mapping with electrocardiographic imaging (ECGI). Circulation 2014;130: 1936-43.

8. Ghanem RN, Burnes JE, Waldo AL, Rudy $Y$. Imaging dispersion of myocardial repolarization, II: noninvasive reconstruction of epicardial measures. Circulation 2001:104:1306-12.

9. Martin RO, Pilkington TC. Unconstrained inverse electrocardiography: epicardial potentials. IEEE Trans Biomed Eng 1972;19:276-85.
10. Oster HS, Taccardi B, Lux RL, Ershler PR Rudy Y. Noninvasive electrocardiographic imaging: reconstruction of epicardial potentials, electrograms, and isochrones and localization of single and multiple electrocardiac events. Circulation 1997:96:1012-24.

11. Ghanem RN, Jia $P$, Ramanathan $C$, Ryu $K$ Markowitz A, Rudy Y. Noninvasive electrocardiographic imaging (ECGI): comparison to intraoperative mapping in patients. Heart Rhythm 2005;2:339-54.

12. Cuculich PS, Wang $Y$, Lindsay $B D$, et al. Noninvasive characterization of epicardial activation in humans with diverse atrial fibrillation patterns. Circulation 2010;122:1364-72.

13. Sapp JL, Dawoud F, Clements JC, Horáček BM Inverse solution mapping of epicardial potentials: quantitative comparison with epicardial contact mapping. Circ Arrhythm Electrophysiol 2012;5: 1001-9.

14. Erem B, Brooks DH, van Dam PM, Stinstra JG MacLeod RS. Spatiotemporal estimation of activation times of fractionated ECGs on complex heart surfaces. Conf Proc IEEE Eng Med Biol Soc 2011:5884-7.

15. Ghosh S, Rudy Y. Accuracy of quadratic versus linear interpolation in noninvasive Electrocardio graphic Imaging (ECGI). Ann Biomed Eng 2005: 1187-201.

16. Revishvili AS, Wissner $E$, Lebedev DS, et al. Validation of the mapping accuracy of a novel non-invasive epicardial and endocardial electrophysiology system. Europace 2015;17:1282-8.

17. Erkapic D, Greiss H, Pajitnev D, et al. Clinical impact of a novel three-dimensional electrocardiographic imaging for non-invasive mapping of ventricular arrhythmias-a prospective randomized trial. Europace 2015;17:591-7.
18. Cuculich $P$, Schill M, Kashani $R$, et al. First report of entirely noninvasive stereotactic cardiac ablation radiotherapy (NO-SCAR) for VT in humans. Heart Rhythm 2016;13:S138.

19. Ramanathan C, Jia $P$, Ghanem $R$, Calvetti $D$, Rudy Y. Noninvasive electrocardiographic imaging (ECGI): application of the generalized minimal residual (GMRes) method. Ann Biomed Eng 2003;31:981-94.

20. Wang $Y$, Rudy Y. Application of the method of fundamental solutions to potential-based inverse electrocardiography. Ann Biomed Eng 2006;34: $1272-88$

21. Oster HS, Rudy Y. Regional regularization of the electrocardiographic inverse problem: a model study using spherical geometry. IEEE Trans Biomed Eng 1997;44:188-99.

22. Pullan AJ, Cheng LK, Nash MP, Ghodrati $A$ MacLeod R, Brooks DH. The inverse problem of electrocardiography. In: Macfarlane PW, van Oosterom A, Pahlm O, Kligfield $\mathrm{P}$, Janse $\mathrm{M}$, Camm J, editors. Comprehensive Electrocardiology. London: Springer, 2010:299-344.

23. Van Oosterom A. A comparison of electrocardiographic imaging based on two source types. Europace 2014;16 Suppl 4:iv120-8.

24. Bear LR, Cheng LK, LeGrice IJ, et al. The forward problem of electrocardiography: is it solved? Circ Arrhythm Electrophysiol 2015;8:677-84.

KEY WORDS cardiac electrophysiology, electrocardiography, inverse problem of electrocardiography, noninvasive electrocardiographic imaging

APPENDIX For a supplemental methods section as well as tables and figures, please see the online version of this article. 